\title{
Quand le sentiment antivaccin mène à la violence : émeute antivaccination de 1885 à Montréal
}

- Citation : CMAJ 2021 April 6;193:E490-2. doi : 10.1503/cmaj.202820-f

Voir la version anglaise de l'article ici : www.cmaj.ca/lookup/doi/10.1503/cmaj.202820

e 30 janvier 2021, des manifestants antivaccins ont brièvement perturbé les efforts de vaccination déployés au Dodger Stadium pour lutter contre le coronavirus du syndrome respiratoire aigu sévère 2 (SRASCoV-2), le virus à l'origine de la maladie à coronavirus 2019 (COVID-19). D'autres manifestants ont pris d'assaut des centres commerciaux pour protester contre le port obligatoire du masque, les couvre-feux et les mesures de confinement qui ont été mis en place pour ralentir la propagation du virus, et au moins une manifestation a forcé la fermeture d'un hôpital ${ }^{1}$. Au cours de la dernière année, on a assisté à une forte croissance des groupes antivaccins sur les médias sociaux ${ }^{2}$. Des manifestations semblables contre les mesures de santé publique liées à la COVID-19, dont certaines ont pris une tournure violente, se sont déroulées dans de nombreuses villes en 2020 et 2021, souvent en raison de clivages politiques partisans ${ }^{3,4}$. Les manifestations violentes contre les campagnes de vaccination ne sont toutefois pas uniques à notre époque, comme en témoigne l'émeute antivaccination de 1885 à Montréal.

Le dernier cas de variole endémique au Canada remonte à 1946, mais avant la vaccination généralisée, les épidémies de variole étaient courantes. Cette maladie mortelle spontanément résolutive était causée par les virus de la variole majeure et de la variole mineure. Elle se propageait par les gouttelettes respiratoires, et l'infection des glandes sébacées provoquait l'apparition de pustules qui éclataient, laissant des cicatrices déprimées chez 65\%-80\% des patients. Le taux de mortalité était d'environ $15 \%$ chez les personnes non vaccinées et non variolisées infectées par la variole ${ }^{5}$. Les survivants développaient une immunité.

En mars 1885, un conducteur de la compagnie de chemin de fer du Grand Tronc (Grand Trunk Railway) porteur de la variole arrive à Montréal; la maladie se répand alors rapidement dans la ville ${ }^{6}$. Les efforts de vaccination déployés par la suite ne se déroulent pas sans heurts. Après l'apparition de plusieurs cas d'érysipèle après la vaccination (proba- blement causés par des conditions insalubres), les responsables de la santé concluent que le lot de vaccins utilisé serait responsable. Ces événements conduisent, en mai 1885, à une suspension du programme de vaccination pendant 3 mois. Les personnes qui s'opposent à la vaccination font courir le bruit que le vaccin antivariolique est dangereux et arrachent les panneaux de mise en garde installés par les travailleurs de la santé publique aux domiciles des personnes ayant reçu un diagnostic de variole. La maladie se propage dans les quartiers pauvres et majoritairement francophones de la ville; les éditoriaux

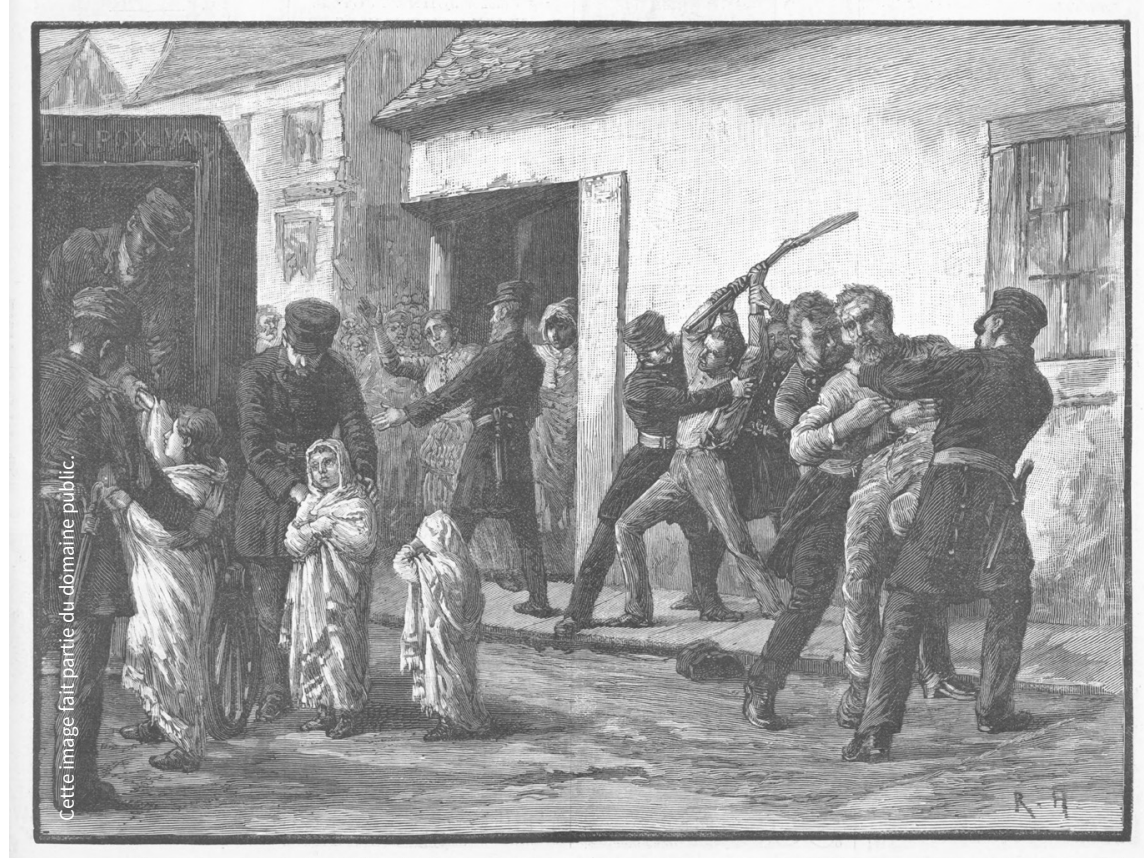

Ce dessin de Robert Harris intitulé «An incident of the smallpox epidemic in Montreal » illustre la violence employée par la police sanitaire pour isoler du public les patients atteints de la variole durant les émeutes antivaccination de 1885. 
anglophones contemporains dénoncent les conditions de vie des taudis habités par les pauvres, ignorant la qualité inégale et souvent médiocre du programme de vaccination. Au cours de l'été, l'épidémie s'aggrave.

Le 2 septembre 1885, le conseil de santé de Montréal estime qu'il y a environ 2000 cas de variole dans la ville. Le 28 septembre, certains experts estiment à 4000 le nombre de cas. À la fin du mois de septembre, la police sanitaire commence à expulser les personnes dont les conditions de logement rendent l'isolement impossible, en utilisant souvent des mesures dures qui isolent les pauvres du logement et du soutien social. Toujours en date du 28 septembre, le conseil de santé décrète que la vaccination sera rendue obligatoire. En réaction, le soir même, une "meute hurlante » encercle le bureau de santé de la partie Est de la ville et " saccage» l'édifice ${ }^{6}$. Les manifestants, aussi préoccupés par les questions d'identité que par la vaccination, crient des phrases comme "Vive les Canadiens français! Les Anglais et les Irlandais ne sont plus les bienvenus à Montréal!? ».

La foule traverse la ville, chassant les policiers appelés en renfort et fracassant les fenêtres des pharmacies qui vendent des vaccins ainsi que des maisons des agents de santé. Les manifestants antivaccins brisent toutes les fenêtres du poste de police central. Le chef de la police est poignardé et lapidé, mais il survit à ses blessures. Les émeutiers tirent sur les policiers, lesquels ont recours à d'autres tactiques pour tenter de disperser la foule. Armés de fusils et de baïonnettes, ils font feu au-dessus des têtes des manifestants. Ils finissent par frapper les émeutiers à coups de matraque, amenant ces derniers à se séparer en plus petits groupes qui continuent à détruire des biens et à endommager des bâtiments.

Le lendemain, les autorités craignent que d'autres événements violents se produisent. Bien que le conseil de santé soit ambivalent quant au recours à la force, 1400 militaires armés se rassemblent à l'hôtel de ville et patrouillent dans la ville. Des revolvers sont distribués à la police sanitaire. Le conseil de santé attribue les émeutes aux mensonges proférés par les militants antivaccins, selon qui les vaccinateurs entreraient dans les chambres à coucher des femmes et attacheraient les enfants pour les vacciner. Ultimement, aucun autre incident violent majeur ne s'est produit. On ne sait pas si l'usage de la force s'est avéré efficace, si l'intérêt pour les émeutes s'est amoindri ou si la foule n'était tout simplement pas organisée. Dans l'ensemble, l'émeute a seulement entraîné des dommages matériels.

Au cours des 5 semaines précédant le 31 octobre, 1391 personnes meurent de la variole à Montréal. Parmi elles, 1286 sont francophones et environ $90 \%$ sont des enfants de moins de 10 ans. Au total, l'épidémie entraîne au moins 19905 cas et 5964 décès dans la province de Québec, dont 3259 dans la seule région de Montréal ${ }^{8}$.

L'émeute n'a pas donné lieu à une capitulation face aux demandes des militants antivaccins ni à une application rigoureuse des politiques. En revanche, après cette épidémie, les opposants à la vaccination se tournent vers les tribunaux et les organisations militantes. En 1887, l'Ontario adopte une loi sur la vaccination, exigeant que "les parents fassent vacciner leurs enfants contre la variole dans les 3 mois suivant leur naissance et que ces derniers soient vaccinés à nouveau tous les 7 ans, au besoin ». Cette loi permet aux villes d'émettre des ordonnances de vaccination en cas d'épidémie de variole et aux commissions scolaires d'exiger un certificat de vaccination pour les élèves ${ }^{9}$. La Ligue antivaccination du Canada, qui s'inspire de la rhétorique et des méthodes des groupes antivaccins britanniques, est créée en 1900 , en partie en réaction à la loi sur la vaccination. La British Anti-Compulsory Vaccination League, fondée en 1867, organise sa propre marche (pacifique) à Leicester en 1885; environ 100000 personnes y participent ${ }^{10}$.

Les militants antivaccins invoquent comme arguments des inquiétudes quant aux libertés individuelles et la crainte que les risques de la vaccination l'emportent sur les bienfaits. À l'époque, les vaccins antivarioliques, qui conte- naient de la lymphe prélevée dans des pustules de vaches ou d'humains infectés, pouvaient être contaminés et causer des infections secondaires ${ }^{11}$. Les opposants aux vaccins se demandaient pourquoi certaines personnes tombent malades après avoir été vaccinées ${ }^{12}$. Les médecins se posaient des questions théoriques sur le mode d'action des vaccins, et beaucoup se demandaient si la variole des vaches, utilisée pour fabriquer les vaccins, était causée par le même virus que la variole ${ }^{13}$.

Fait notable pour les médecins d'aujourd'hui : ce n'est pas seulement l'incertitude scientifique qui a permis aux militants antivaccins de lutter efficacement contre l'adoption des lois de santé publique. En 1906, soit 2 décennies après les émeutes de Montréal, la Ligue antivaccination du Canada réussit à convaincre le service de la santé de Toronto de supprimer l'obligation de vaccination dans les écoles. Les militants antivaccins inscrivent alors leur opposition dans un contexte de classes sociales et d'autonomie personnelle. Alexander Ross, l'un des fondateurs de la Ligue, écrit en 1888 : "Ce sont les femmes et les enfants pauvres des travailleurs, ce sont les employés des magasins et les ouvriers des usines et des ateliers, ce sont les travailleurs et les travailleuses qui sont menacés et pourchassés par les mercenaires de la loi infâme sur la vaccination obligatoire ${ }^{14}$. " L'opposition à la vaccination antivariolique, qu'elle soit exprimée par des foules en colère ou seulement dans des salles d'audience, traduit un malaise face à un transfert de pouvoir concernant une décision personnelle en matière de santé.

Bien que la variole ait fini par être éradiquée, le sentiment antivaccin est bien enraciné. Selon des études récentes, près d'un tiers des Canadiens ne veulent pas se faire vacciner contre le SRAS-CoV- $2^{15}$. Aujourd'hui, tout comme en 1885 , de nombreuses personnes considèrent les politiques de santé publique comme une autre expression des déséquilibres de pouvoir dans la société. S’il y a une leçon que les médecins doivent tirer de l'émeute antivaccination qui a eu lieu à 
Montréal, c'est sans doute la nécessité de respecter le pouvoir de chacun de prendre ses propres décisions en matière de santé et de se rappeler que ce pouvoir peut prendre de multiples formes. De nombreuses personnes ont besoin de temps pour prendre une décision personnelle en matière de santé, et elles le font souvent dans le cadre d'une relation patient-médecin de confiance.

\section{Jonathan M. Berman PhD}

Département des sciences fondamentales, College of Osteopathic Medicine du New York Institute of Technology à l'Université de l'Arkansas, à Jonesboro, en Arkansas.

\section{Références}

1. KOIN 6 news staff. ClarkCo: 'Anti-vax' protesters force lockdown at ClarkCo hospital. KOIN 6 News [Portland (OR)] 2021 Jan. 29. (consulté le 20 févr. 2021).

2. Burki T. The online anti-vaccine movement in the age of COVID-19. Lancet Digit Health 2020;2: e504-5.
3. Hapuhennedige S. Public health experts are learning from Canada's anti-mask protests. CMAJ 2020;192:E1274-5.

4. Hotez PJ. COVID19 meets the antivaccine movement. Microbes Infect 2020;22:162-4.

5. Gani R, Leach SJN. Transmission potential of smallpox in contemporary populations [erratum publié dans Nature 2002;415:1056]. Nature 2001;414:748-51.

6. A vaccination riot in Montreal. The Deseret News 1885 Oct. 2; By Telegraph:1(col 1).

7. Bliss M. Plague: a history of smallpox in Montreal. Toronto: HarperCollins; 1991.

8. MacDougall H. Be wise - immunize! Vaccination in Canada, 1798-1978. Can Pharm J 2007;140: S5-S7.

9. Arnup K. "Victims of vaccination?": Opposition to compulsory immunization in Ontario, 1900-90. Can Bull Med Hist 1992;9:159-76.

10. Wolfe RM, Sharp LKJB. Anti-vaccinationists past and present. BMJ 2002;325:430-2.

11. Durbach N. Bodily matters: the anti-vaccination movement in England, 1853-1907. Durham (NC): Duke University Press; 2005.

12. Keelan J, Risk, efficacy and viral attenuation in debates over smallpox vaccination in Montreal, 1870. In: Crafting immunity: working histories of clinical immunology. Kroker K, Mazumdar MH, Keelan JE, editors. Burlington (VT): Ashgate; 2008:29-41.

13. Farley M, Keating $P$, Keen $O$, et al. Vaccination in Montreal in the second half of the 19th century: practices, obstacles and resistance [chapitre en français]. In: Sciences \& médecine au Québec: perspectives sociohistoriques. Québec: Institut québécois de recherche sur la culture; 1987:87-127.

14. Rutty CJ. Book review: Heather MacDougall, Activists and Advocates: Toronto's Health Department, 1883-1983, Toronto and Oxford, Dundurn Press, 1990. Pp 297, endnotes, selected bibliography, 80 illustrations. ISBN 1-55002-072-2, \$29.95 (cloth). Scientia Canadensis 1992;16:109-12.

15. Bricker D. Many Canadians aren't in a hurry to receive COVID-19 vaccine. https://www.ipsos. com/en-ca/news-polls/many-canadians-arent -in-a-hurry-to-receive-covid-19-vaccine. Ipsos 2020 Nov. 6 (consulté le 10 déc. 2020).

Cet article a été révisé par des pairs.

Intérêts concurrents : Aucun déclaré.

Propriété intellectuelle du contenu : Il s'agit d'un article en libre accès distribué conformément aux modalités de la licence Creative Commons Attribution (CC BY-NC-ND 4.0), qui permet l'utilisation, la diffusion et la reproduction dans tout médium à la condition que la publication originale soit adéquatement citée, que l'utilisation se fasse à des fins non commerciales (c.-à-d., recherche ou éducation) et qu'aucune modification ni adaptation n'y soit apportée. Voir : https://creativecommons.org/ licenses/by-nc-nd/4.0/deed.fr. 